\title{
Expression Analysis of MicroRNA-196a in Esophageal Cancer
}

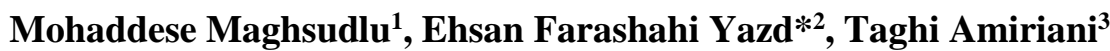

1. Department of Genetics, Faculty of Medicine, Shahid Sadoughi University of Medical Sciences, Yazd, Iran ORCID ID: 0000-0003-3586-5429. m.maghsudlu.2@gmail.com

2. Stem Cell Biology Research Center, Yazd Reproductive Science Institute, Shahid Sadoughi University of Medical Sciences, Yazd, Iran ORCID ID: 0000-0003-1122-0626. ehsanfarashahi@gmail.com

3. Sayad Shirazi Hospital, Golestan Center of Gastroenterology and Hepatology, Golestan University of Medical Sciences, Gorgan, Iran

\begin{abstract}
Backgrounds and objectives: Esophageal squamous cell carcinoma (ESCC) is a prevalent subtype of esophageal cancer in certain areas of the world. Molecular evaluations can provide a better understanding of the disease that could contribute to earlier diagnosis and treatment. Amplification or absence of microRNA (miRNA) genes has been described in many types of cancer, indicating that altered patterns of miRNA expression may affect cell cycle and its features. In this study, we investigated alteration of miR-196a expression in ESCC tissues and its association with tumor status.

Methods: The study was done on 30 specimens including matched tumor and normal tumor margin tissues. RNA extraction and complementary DNA synthesis were performed using specific primers. Quantitative PCR was done to assess the relative expression of miR-196a in tumor tissues of patients with ESCC. Data were normalized by expression level of SNORD gene as an internal control. The GraphPad Prism and SPSS software were used for analysis of data.

Results: MiR-196a expression was higher in cancer tissues compared to normal tissues $(\mathrm{P}>0.05)$. The miRNA upregulation had no significant correlation with clinicopathological features such as age, gender and survival rate.

Conclusion: The results of the present study indicate a nonsignificant increased expression of miR196a in ESCC tumor specimen. Future studies with a larger sample size could confirm our findings. Keywords: Esophageal squamous cell carcinoma; gene expression; microRNA; miR-196a
\end{abstract}

\section{$\begin{array}{lll}\text { Received: 2019/08/20 } & \text { Revised:2019/09/03 } & \text { Published:2019/10/03 }\end{array}$}

Correspondence: Ehsan Farashahi Yazd, Research Center, Yazd Reproductive Science Institute, Shahid Sadoughi University of Medical Sciences, Yazd, Iran

Tel: +98-3538247086

Email: ehsanfarashahi@ssu.ac.ir 


\section{INTRODUCTION}

Esophageal cancer (EC) is one of the most prominent cancers in the world. Esophageal squamous cell carcinoma (ESCC) is the common histopathological type of EC, which is diagnosed by an early-stage lymph node metastasis (1-3). ESCC accounts for $90 \%$ of EC cases in the Asian EC belt that extends from Northern Iran to the North-Central China. Diagnosis of EC in the late stages and when the symptoms appear makes treatment usually ineffective. Therefore, it is imperative to seek reliable biomarkers for an early detection and prognosis of ESCC (4).

MicroRNAs (miRNAs) are a class of small non-coding, single stranded RNAs of 17-25 ribonucleotides, which are known as important regulators of gene expression (5). Binding of these molecules to 3'-untranslated region of target mRNAs leads to mRNA degradation or translational repression (6). Studies show that miRNAs are involved in numerous cellular pathways related to differentiation, proliferation, apoptosis and metabolism, and are also important for regulation of tumor suppressor genes in many cancers (7). MiRNA dysregulation has been detected in different types of primary and metastatic cancer $(8,9)$. Several studies have investigated this dysregulation in malignancies of the gastrointestinal tract (10). Among miRNAs that play a role in various stages of cancer, miRNA-196 family can be considered as tumor status markers, especially in malignancies with squamous cell origin and cancers of digestive system. MiR-196a belongs to the mentioned family and its genes are located on chromosomes 12 and 17 in the homeobox gene clusters (Hox) regions. Both genes transcribe the same functional mature miRNA sequence. Evidence suggests that expression of this microRNA may be altered in esophageal adenocarcinoma cancer cells. Moreover, it's up regulation has been recently discovered in the saliva specimens from ESCC patients and ESCC cell lines $(11,12)$. It seems that the level of miR-196a expression is associated with metastasis, poor prognosis and reduced overall survival of the patients $(13,14)$. Therefore, in the present study, we

evaluated the relative expression level of miR-196a in cancer and normal margin esophagus tissues. Moreover, we investigated whether altered expression of this miRNA was associated with the patient's short-term survival.

\section{MATERIALS AND METHODS Sample collection}

Overall 30 ESCC and normal tissue specimens were taken from 15 patients (7 females and 8 males, mean age 71 years) who had undergone endoscopy. Written consent was obtained from all patients prior to sampling. The samples were only collected from patients with squamous cell carcinoma and those with adenocarcinoma were excluded. Moreover, patients with recurrent disease and those receiving chemotherapy were not included in the study. Considering the limited sampling time and large number of excluded subjects, the number of samples was not large. The patients were followed up for 6 months. The study was approved by the ethics committee of Shahid Sadoughi University of Medical Sciences, Yazd, Iran.

Fresh samples were soaked in RNA Later (Qiagen, Hilden, Germany) and kept at -80 ${ }^{\circ} \mathrm{C}$.

\section{RNA extraction, cDNA synthesis and qPCR}

Total RNA was extracted from the tissue samples using the RNA RiboEx extraction kit (GeneAll). Then, cDNA was synthesized using the MicroRNA $1^{\text {st }}$ Strand cDNA Synthesis Kit (Stem cell Tech, Tehran, Iran) and the polyA tailing method was used. To form the polyA tails, the samples were incubated at $37^{\circ} \mathrm{C}$ for 30 minutes. Next, they were incubated at $60{ }^{\circ} \mathrm{C}$ for 20 minutes for polyA polymerase inactivation. Finally, the reverse transcription step was carried out on polyadenylated samples by incubation at 25 ${ }^{\circ} \mathrm{C}$ for 10 minutes, at $42{ }^{\circ} \mathrm{C}$ for 60 minutes and at $70{ }^{\circ} \mathrm{C}$ for 10 minutes. RT-qPCR was 
done using $1 \mu \mathrm{L}$ cDNA, microRNA QPCR master mix (Stem cell Tech, Tehran, Iran) and commercial miR-specific primers with the following sequences: forward primer for miR196a (ATT GAT GCG TAG GTA GTT TCA), and the reverse universal primer (Stem cell Tec, Tehran, Iran). The SNORD gene was used as an internal control and the forward primer sequence was AGA TTT AAC AAA AAT TCG TCA C. RT-PCR was performed in a Rotor Gene real-time quantitative PCR instrument (Qiagen, Germany) under the following conditions: $95^{\circ} \mathrm{C}$ for two minutes, followed by 40 cycles of $95{ }^{\circ} \mathrm{C}$ for five seconds and $60{ }^{\circ} \mathrm{C}$ for 30 seconds. All RTqPCR amplifications were carried out in duplicate, and a negative control was placed for each reaction for quality control. Finally, accuracy of the amplified RT-qPCR products was determined by assessing presence of single, sharp melting curves.

\section{Statistical analysis}

Data obtained from the RT-PCR experiment were analyzed using the GraphPad Prism 6 software (GraphPad Software Inc., San

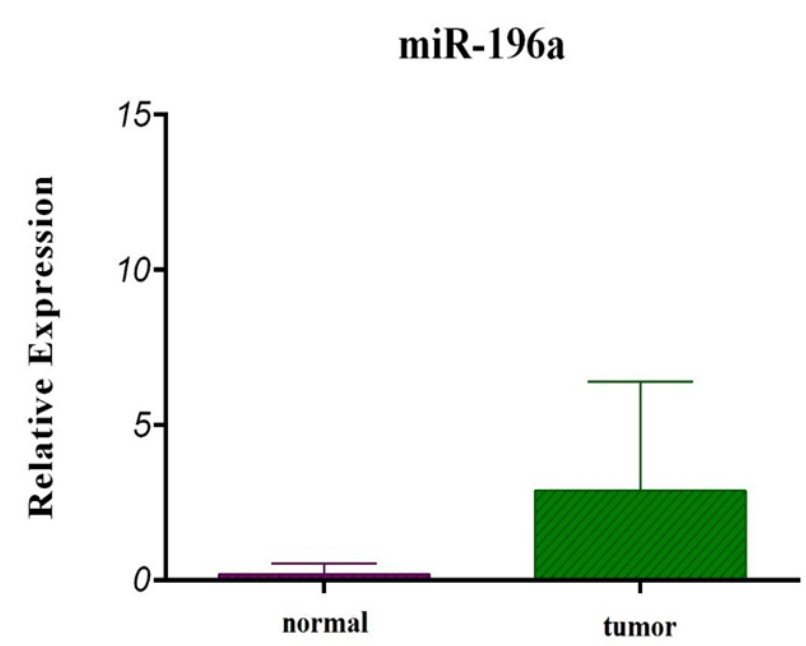

Diego, USA) and SPSS16 software (SPSS Inc., Chicago, IL). Level of miRNA expression in each sample was normalized to that of the SNORD gene. Then, miRNA expression level in the tumor samples and normal samples was compared using the $2^{-}$ $\Delta \Delta \mathrm{Ct}$ method. Normal distribution of the data was confirmed by the Kolmogorov-Smirnov test. Comparisons were made between the two groups using t-test at significance of 0.05 . The Chi-square test and logistic regression analysis were used to evaluate the relationship between miRNA expression and clinicopathological characteristics of ESCC patients.

\section{RESULTS}

\section{MiR-196a was slightly upregulated in ESCC tumors}

The relative expression of miR-196a in tumor tissues was slightly higher $(3.2 \pm 6.3)$ than in normal adjacent tissues $(\mathrm{P}=0.488)$ (Figure1A). Figure 1B shows the melting curve of final products amplified by miR196a primers.
B)

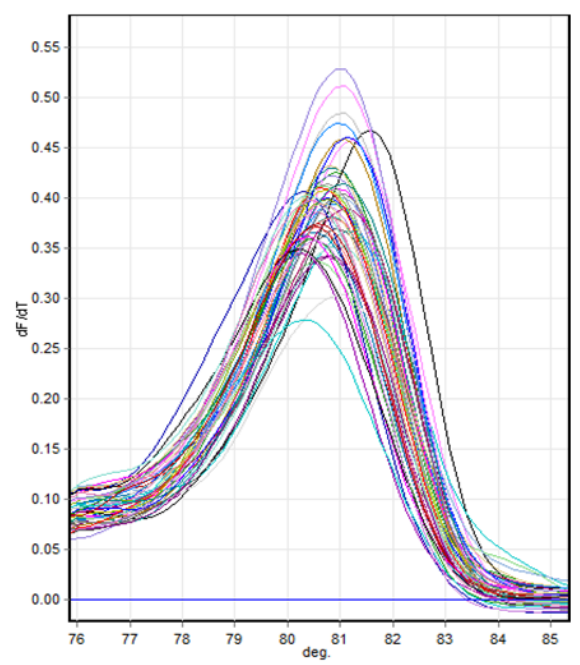

Figure 1. Specific gene expression analysis of miR-196a. A) Relative expression of miR-196a in tumor and tumor margin samples. B) Melting curve of the final product replicated with miR-196a primers. 
Journal of Clinical and Basic Research (JCBR). 2019; 3(3): P 5-11.

\section{Correlation analysis of the miR-196a with clinicopathological features of ESCC patients}

Expression level of miR-196a was categorized into high and low groups according to the median value; however, no significant correlation was noted between miR-196a expression and patients' clinical characteristics (Table 1).

Table 1. The relationship between miR-196a expression and clinical characteristics of patients with

\begin{tabular}{|c|c|c|c|c|c|}
\hline \multicolumn{6}{|c|}{ ESCC } \\
\hline \multirow{3}{*}{ Clinical profile } & \multirow{3}{*}{ Variable } & & miR-196a & miR-196a & \multirow{3}{*}{ P-value } \\
\hline & & Potal mumiter & overexpression & underexpression & \\
\hline & & 15 & 8 & 7 & \\
\hline \multirow[t]{2}{*}{ Age } & $>70$ years & 6 & 4 & 2 & \multirow{2}{*}{0.257} \\
\hline & $<70$ years & 9 & 4 & 5 & \\
\hline \multirow[t]{2}{*}{ Sex } & Male & 8 & 3 & 5 & \multirow{2}{*}{0.16} \\
\hline & Female & 7 & 5 & 2 & \\
\hline \multirow{2}{*}{ Survival } & $>6$ months & 6 & 2 & 4 & \multirow{2}{*}{0.130} \\
\hline & $<6$ months & 9 & 6 & 3 & \\
\hline
\end{tabular}

\section{.DISCUSSION}

Recently, extensive research has been performed on the role of miRNAs expression in cell function, survival and development. Investigating miRNAs expression patterns in different cancers can be also beneficial (15). MiRNAs have been suggested as potential biomarkers and therapeutic targets for various diseases (16).

MiR-196a is involved in normal development and the pathogenesis of diseases, such as cancer. This miRNA is capable of regulating different genes in various cell types and under different statuses. Although located in the Hox gene cluster, miR-196a regulation can be also modulated by factors such as circular RNAs in oral squamous cell carcinoma. Thus, further research is required to clarify the regulation of miR-196a in different tissues, including EC (17). Regarding the regulatory function of microRNAs in cells, determining their putative targets could reveal the outcome of expression alterations in normal and affected tissues. Amongst all putative targets for miR-196 that have been found by in silico approaches, Hox mRNAs are preferentially targeted by the miR-196 gene family which are associated with malignancies, such as gastrointestinal tumors. Other significant targets thought to be involved in different cancers include, $\mathrm{p}^{27 \mathrm{kip}}$ in gastric cancer (18), NFKBA in pancreatic cancer (19), NME4 in oral cancer (20) and Annexin A1 in head and neck and esophageal adenocarcinoma (21). Considering tissue similarities, Annexin A1 and NME4 seem to be appropriate candidates for future research on ESCC. A recent study on Annexin A1 in Het-1A cell line of human normal esophageal epithelial and the EC109 cell line of ESCC indicated that miR-196a may affect different features of ESCC cells such as proliferation, invasion and migration by targeting ANXA1. The study also stated that the Annexin A1-induced regulation of MMP-2, COX2 and E-cadherin may contribute to the development of cancer cell characteristics. Thus, the miR-196a/ANXA1 axis may be considered a potential therapeutic target in ESCC (22). 
Overexpression of miR-196a is common in digestive tract malignancies such as oral, gastric, colon and colorectal cancers and correlates with poor prognosis $(13,14,23)$. In a recent study on gastric cancer stem cells, miR-196a was remarkably upregulated in less-differentiated and more invasive gastric cancer cells (24). A study on head and neck squamous cell carcinoma revealed that downexpression of miR-196a with anti-miR reduced invasion and migration of tumor cells as well as fibronectin adhesion (25). Despite the strong evidence on the oncogenic function of miR-196 in cancers, few reports have indicated that the miR-196 family can act as tumor suppressors in some cancers, such as melanoma and breast cancer (26). Some studies have suggested miR-196a as a noninvasive biomarker for diagnosis, staging and prognosis of cancers (27-29).

Correlation of aberrant miR-196a expression and single nucleotide polymorphisms with chemosensitivity and radioresistance has been demonstrated in different studies, suggesting that investigating expression patterns and known polymorphisms might be useful in development of therapeutic strategies (29, 30).

Upregulation of miR-196a was previously shown in esophageal adenocarcinoma, in saliva specimens and ESCC cell lines $(11,12$, 22). The alteration of miR-196a expression is in concordance with previous studies, but the lack of statistical significance as well as the non-significant relationship between expression changes and short survival could be due to the small sample size in our study.

\section{CONCLUSION}

Overall, the present study shows that miR196a expression is altered in ESCC specimens. Considering the important role of this miRNA in cancers of the upper gastrointestinal tract and its capability as a potential biomarker, further investigations with a larger sample size and on serum samples could be beneficial.

\section{ACKNOWLEDGMENTS}

We are grateful to the Golestan Research Center of Gastroenterology and Hepatology for their contribution in sample collection.

\section{DECLARATIONS Funding}

This research has been supported by the Shahid Sadoughi University of Medical Sciences, Iran.

\section{Ethics approvals and consent to participate}

The study was approved by the ethics committee of Shahid Sadoughi University of Medical Sciences (approval code: IR.SSU.MEDICINE.REC.1395.194). Written informed consent was obtained from all participants prior to sampling. All procedures were performed in accordance with the ethical standards of the responsible committee on human experimentations (institutional and national) and with the declaration of Helsinki.

\section{Conflict of interest}

The authors declare that there is no conflict of interest regarding the publication of this article.

\section{REFERENCES}

1. Lin D-C, Hao J-J, Nagata Y, Xu L, Shang L, Meng $X$, et al. Genomic and molecular characterization of esophageal squamous cell carcinoma. Nature Genet. 2014;46(5):467. [DOI:10.1038/ng.2935]

2. Chen W, Sun K, Zheng R, Zeng H, Zhang S, $\mathrm{Xia} C$, et al. Cancer incidence and mortality in China, 2014. Chinese journal of cancer research. 2018;30(1): 1 . [DOI:10.21147/j.issn.10009604.2018.01.01]

3. Fukuhara $\mathrm{T}$, Hiyama $\mathrm{T}$, Tanaka $\mathrm{S}$, Oka $\mathrm{S}$, Yoshihara M, Arihiro K, et al. Characteristics of esophageal squamous cell carcinomas and lugolvoiding lesions in patients with head and neck squamous cell carcinoma. J Clin Gastroenterol. 2010;44(2):e27-e33.

[DOI:10.1097/MCG.0b013e3181b31325]

4. Abnet CC, Arnold M, Wei W-Q. Epidemiology of esophageal squamous cell carcinoma. Gastroenterology. 2018;154(2):360-73. [DOI:10.1053/j.gastro.2017.08.023] 
5. Cho WC. OncomiRs: the discovery and progress of microRNAs in cancers. Mol Cancer. 2007;6(1):60. [DOI:10.1186/1476-4598-6-60]

6. Valencia-Sanchez MA, Liu J, Hannon GJ, Parker R. Control of translation and mRNA degradation by miRNAs and siRNAs. Genes Dev. 2006;20(5):515-24. [DOI:10.1101/gad.1399806]

7. Jamali L, Tofigh R, Tutunchi S, Panahi G, Borhani F, Akhavan S, et al. Circulating microRNAs as diagnostic and therapeutic biomarkers in gastric and ECs. Journal of cellular physiology. 2018;233(11):8538-50. [DOI:10.1002/jcp.26850]

8. Kunej T, Godnic I, Horvat S, Zorc M, Calin GA. Cross talk between microRNA and coding cancer genes. Cancer J. 2012;18(3):223. [DOI:10.1097/PPO.0b013e318258b771]

9. Zaravinos A. The regulatory role of microRNAs in EMT and cancer. J Oncol. 2015;2015(2015):13. [DOI:10.1155/2015/865816] 10. Saito Y, Suzuki H, Hibi T. The role of microRNAs in gastrointestinal cancers. $\mathrm{J}$ Gastroenterol. 2009;44(19):18-22. [DOI:10.1007/s00535-008-2285-3]

11. Luthra R, Singh R, Luthra M, Li Y, Hannah C, Romans A, et al. MicroRNA-196a targets annexin A1: a microRNA-mediated mechanism of annexin A1 downregulation in cancers. Oncogene. 2008;27(52):6667-78.

[DOI:10.1038/onc.2008.256]

12. Fendereski M, Zia MF, Shafiee M, Safari F, Saneie MH, Tavassoli M. MicroRNA-196a as a Potential Diagnostic Biomarker for Esophageal Squamous Cell Carcinoma. Cancer Investigation. 2017;35(2):78-84.

[DOI:10.1080/07357907.2016.1254228]

13. Ge J, Chen Z, Li R, Lu T, Xiao G. Upregulation of microRNA-196a and microRNA$196 \mathrm{~b}$ cooperatively correlate with aggressive progression and unfavorable prognosis in patients with colorectal cancer. Cancer Cell Int. 2014;14(1):128. [DOI:10.1186/s12935-014-01282]

14. Lu Y-C, Chang JT, Liao C-T, Kang C-J, Huang S-F, Chen I-H, et al. OncomiR-196 promotes an invasive phenotype in oral cancer through the NME4-JNK-TIMP1-MMP signaling pathway. Mol Cancer. 2014;13(1):218. [DOI:10.1186/1476-4598-13-218]

15. Rupaimoole R, Slack FJ. MicroRNA therapeutics: towards a new era for the management of cancer and other diseases. Nature reviews Drug discovery. 2017;16(3):203. [DOI:10.1038/nrd.2016.246]
16. Huang J, Zhang SY, Gao YM, Liu YF, Liu YB, Zhao ZG, et al. MicroRNAs as oncogenes or tumour suppressors in oEC: potential biomarkers and therapeutic targets. Cell Prolif. 2014;47(4):277-86. [DOI:10.1111/cpr.12109]

17. Wang L, Wei Y, Yan Y, Wang H, Yang J, Zheng Z, et al. CircDOCK1 suppresses cell apoptosis via inhibition of miR-196a-5p by targeting BIRC3 in OSCC. Oncology reports. 2018;39(3):951-66. [DOI:10.3892/or.2017.6174]

18. Sun M, Liu X, Li JH, Yang JS, Zhang EB, Yin DD, et al. MiR-196a Is Up-regulated in Gastric cancer and Promotes Cell proliferation by Downregulating p27kip1. Molecular cancer therapeutics. 2012:molcanther. 1015.2011. [DOI:10.1158/1535-7163.MCT-11-1015]

19. Huang $F$, Tang J, Zhuang $X$, Zhuang $Y$, Cheng W, Chen W, et al. MiR-196a promotes pancreatic cancer progression by targeting nuclear factor kappa-B-inhibitor alpha. PloS one. 2014;9(2):e87897.

[DOI:10.1371/journal.pone.0087897]

20. Lu Y-C, Chang JT, Liao C-T, Kang C-J, Huang S-F, Chen I-H, et al. OncomiR-196 promotes an invasive phenotype in oral cancer through the NME4-JNK-TIMP1-MMP signaling pathway. Molecular cancer. 2014;13(1):218. [DOI:10.1186/1476-4598-13-218]

21. Rajewsky N, Socci ND. Computational identification of microRNA targets. Genome Biology. 2004;5(2):P5. [DOI:10.1186/gb-2004-52-p5]

22. Hu C, Peng J, Lv L, Wang X, Zhou Y, Huo J, et al. miR 196a regulates the proliferation, invasion and migration of esophageal squamous carcinoma cells by targeting ANXA1. Oncology letters. 2019;17(6):5201-9. [DOI:10.3892/ol.2019.10186]

23. Schimanski CC, Frerichs K, Rahman F, Berger M, Lang H, Galle PR, et al. High miR196a levels promote the oncogenic phenotype of colorectal cancer cells. World J Gastroenterol. 2009;15(17):2089-96.

[DOI:10.3748/wjg.15.2089]

24. Pan Y, Shu X, Sun L, Yu L, Sun L, Yang Z, et al. miR-196a-5p modulates gastric cancer stem cell characteristics by targeting Smad4. International journal of oncology. 2017;50(6):1965-76.

[DOI:10.3892/ijo.2017.3965]

25. Darda L, Hakami F, Morgan R, Murdoch C, Lambert DW, Hunter KD. The role of HOXB9 and miR-196a in head and neck squamous cell 
Journal of Clinical and Basic Research (JCBR). 2019; 3(3): P 5-11.

carcinoma. PloS one. 2015;10(4):e0122285.

[DOI:10.1371/journal.pone.0122285]

26. Lu Y-C, Chang JT, Chan E-C, Chao Y-K, Yeh

T-S, Chen J-S, et al. miR-196, an emerging cancer

biomarker for digestive tract cancers. Journal of Cancer. 2016;7(6):650. [DOI:10.7150/jca.13460]

27. Lu Y-C, Chang JT-C, Huang Y-C, Huang C-

$\mathrm{C}$, Chen W-H, Lee L-Y, et al. Combined determination of circulating miR-196a and miR196b levels produces high sensitivity and specificity for early detection of oral cancer. Clinical biochemistry. 2015;48(3):115-21. [DOI:10.1016/j.clinbiochem.2014.11.020]

28. Hui P, Gysler SM, Uduman M, Togun TA, Prado DE, Brambs CE, et al. MicroRNA signatures discriminate between uterine and ovarian serous carcinomas. Human pathology. 2018;76:133-40.

[DOI:10.1016/j.humpath.2018.02.019]

29. Suh YE, Raulf N, Gäken J, Lawler K, Urbano TG, Bullenkamp J, et al. MicroRNA-196a promotes an oncogenic effect in head and neck cancer cells by suppressing annexin A1 and enhancing radioresistance. International journal of cancer. 2015;137(5):1021-34. [DOI:10.1002/ijc.29397]

30. Schimanski CC, Frerichs K, Rahman F, Berger M, Lang H, Galle PR, et al. High miR196a levels promote the oncogenic phenotype of colorectal cancer cells. World journal of gastroenterology: WJG. 2009;15(17):2089. [DOI:10.3748/wjg.15.2089] 